\title{
La FMH en faveur du tiers garant
}

\author{
Du point de vue du corps médical, le tiers garant est le système de facturation à \\ favoriser dans le secteur ambulatoire (LAMal). Pour tous les acteurs impliqués - \\ patient, médecin et assureur - c'est le système le mieux adapté pour répondre aux \\ exigences en matière de qualité, de coûts et d'efficience de notre système de santé \\ et en même temps pour les promouvoir.
}

\section{Ernst Gähler ${ }^{a}$, \\ Irène Marty ${ }^{b}$, \\ Kerstin Schutz}

a Dr, vice-président de la FMH, responsable du département Tarifs et conventions pour la médecine ambulatoire en Suisse

b FMH, cheffe adjointe de la division Tarifs et conventions pour la médecine ambulatoire en Suisse

c FMH, Tarifs et conventions pour la médecine ambulatoire en Suisse
Correspondance:

FMH / Division Tarifs et conventions pour la médecine ambulatoire en Suisse Froburgstrasse 15

CH-4600 Olten

Tél. 0313591230

Fax 0313591238

tarife.ambulant[at]fmh.ch
La FMH soutient la règle en vigueur conformément à l'art. 42 al. 1 LAMal, selon laquelle le système du tiers garant prédomine dans le secteur ambulatoire, pour autant qu'aucune autre convention n'ait été signée entre les fournisseurs de soins et les assureurs.

Le présent article reprend des extraits du papier de position de la FMH, dont vous trouverez la version complète sur notre site www.fmh.ch

\section{Contexte}

De manière générale, les prestations du secteur ambulatoire prises en charge par l'assurance obligatoire des soins (AOS) sont indemnisées selon deux systèmes de facturation distincts [1].

\section{Tiers garant \\ Le système du tiers garant est celui qui prédo- mine conformément à l'art. 42 al. 1 LAMal. Sauf convention contraire entre les assureurs et les fournisseurs de prestations, l'assuré est le débi- teur de la rémunération envers le fournisseur de prestations. L'assuré a, dans ce cas, le droit d'être remboursé par son assureur. \\ Tiers payant \\ Conformément à l'art. 42 al. 2 LAMal, assureurs et fournisseurs de prestations peuvent convenir que l'assureur est le débiteur de la rémunéra- tion. Il s'agit du système du tiers payant.}

Les deux systèmes de rémunération, le tiers garant et le tiers payant, sont régis par l'art. 42 LAMal [2]. Dans notre pays, le système utilisé majoritairement pour les factures est celui du tiers garant. C'est aussi le système prédominant cité dans la LAMal.

Mais ces dernières années, différents assureurs-maladie sont montés au créneau pour dénoncer ce système de facturation. Ils prétendent qu'avec le tiers payant, et en particulier avec l'introduction de l'échange électronique des données via un intermédiaire, des économies de coûts pourraient être réalisées et répercutées sur les primes.

Depuis pas mal de temps, des différences notables existent entre les assureurs et les médecins concernant l'échange électronique des données. Avec le tiers garant, les factures sont traitées par les centres de confiance des médecins alors qu'avec le tiers payant, les factures sont gérées par l'intermédiaire de MediData, majoritairement financé par les assureurs.

Il est du reste tout à fait compréhensible que les assureurs préfèrent le tiers payant car l'augmentation de leur chiffre d'affaires infléchit le rapport entre chiffre d'affaires et coûts administratifs. Une inflexion dont on ne peut pas dire qu'elle ne soit pas souhaitée de la part des assurances-maladie.

\section{Avantages du tiers garant}

Le tiers payant augmente le volume de factures et les coûts administratifs, ce qui risque de se répercuter sur les primes.

Avec le système du tiers payant, toutes les factures de médecin sont automatiquement et directement adressées aux assurances-maladie. Pour le corps médical, c'est un non-sens économique.

- En effet, comme diverses études l'ont démontré, les cabinets émettent une proportion considérable de petites factures (inférieures à CHF 100.-) qui relèvent de l'assurance obligatoire des soins et que les patients paient souvent directement «de leur poche». Le traitement de toutes ces petites factures selon le système du tiers payant n'entraînerait qu'un surplus de bureaucratie et un surcoût administratif sans aucun avantage médical. Le passage du système du tiers garant à celui du tiers payant dans tout le pays se chiffrerait approximativement à CHF 750 millions.

- Mais aussi, avec le tiers payant, chaque montant supérieur à celui de la franchise est payé par les assureurs avec des répercussions directes sur les primes. Des études réalisées avec santésuisse ont montré qu'une différence de près de $15 \%$ existe entre le nombre de factures effectivement produites et celles envoyées aux assurances pour remboursement. Il faut donc s'attendre à ce que les coûts administratifs, et donc ceux ayant des répercussions sur les primes, subissent une nette augmentation si le système du tiers payant est appliqué à tout le pays. 
- En outre, des projections montrent que si les patients transmettaient tous les «petits» montants à leur assurance-maladie, le volume d'un canton pourrait augmenter de 5 à $8 \%$ ce qui entraînerait la baisse de la valeur du point tarifaire de ce canton.

De cette manière, les coûts administratifs et le chiffre d'affaires des assureurs augmentent sans qu'un quelconque avantage médical ne soit créé pour les assurés.

\section{Le tiers payant concentre plus de pertes sur débiteurs que le tiers garant}

L'évaluation des chiffres de la Caisse des médecins des cantons de Zurich et de Saint-Gall permet de réfuter de manière exemplaire l'argument selon lequel

\section{Figures 1 et 2}

Statistique de la Caisse des médecins, avril 2011- mars 2012, cantons de Zurich et de Saint-Gall.

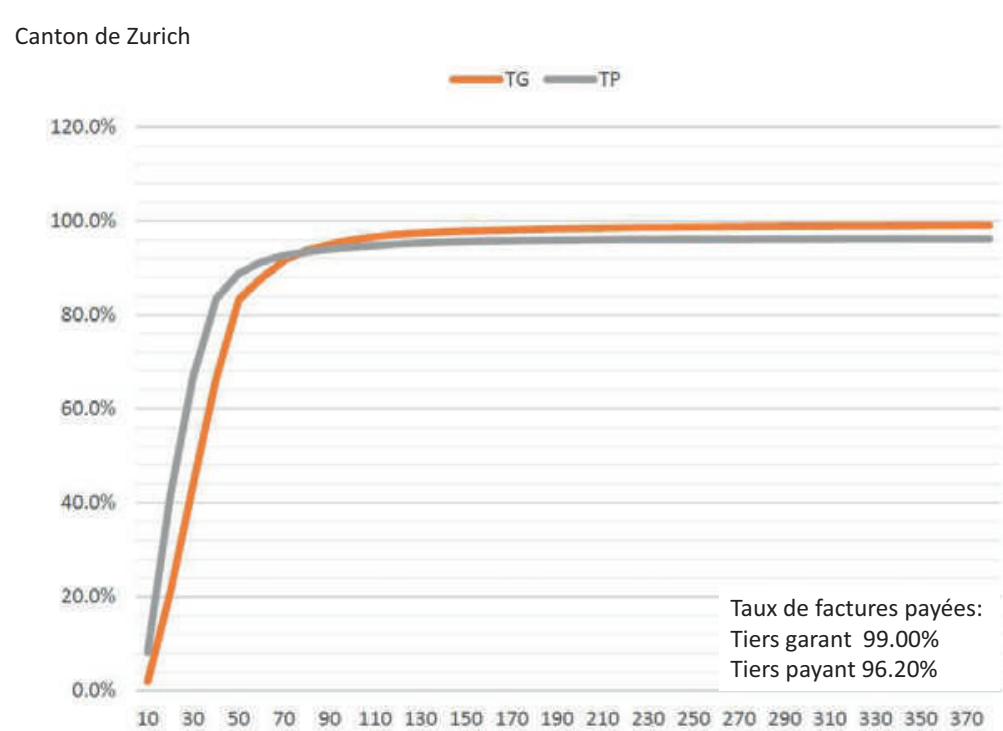

Canton de Saint-Gall

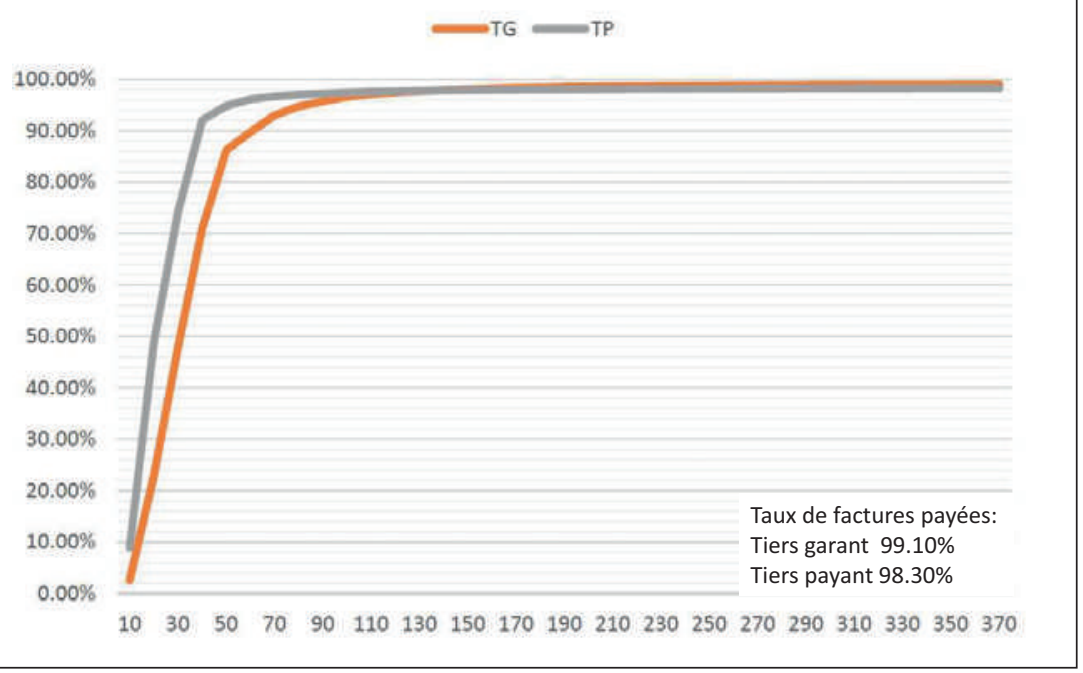

le système du tiers payant assure un meilleur paiement des factures.

Ces chiffres indiquent en effet qu'avec le tiers payant, la proportion de factures payées au terme de 30 jours est moins élevée qu'avec le tiers garant. Dans ces deux cantons, les impayés sont également moins élevés avec le tiers garant.

Les évaluations de plusieurs cantons montrent que les pertes sur débiteurs sont généralement moins importantes avec le tiers garant qu'avec le tiers payant. La répartition des risques est également meilleure avec le tiers garant qu'avec le tiers payant car le nombre de débiteurs (les patients) est naturellement plus élevé et le montant par débiteur moins élevé.

Avec le tiers payant, les dérogations de paiement sont la norme au détriment du patient

Avec le tiers garant, des dérogations de paiement peuvent être accordées dans certains cas appropriés. Notamment lorsqu'un patient doit s'attendre à un traitement long et coûteux, le médecin peut convenir avec lui d'une dérogation [3] pour une durée déterminée dans le but de se préserver de factures élevées. Mais de telles conventions doivent être conclues individuellement en fonction de la situation. Avec le tiers payant, cette pratique serait la norme pour tous les patients et pour toutes les factures au détriment de la protection des données et du pouvoir de décision des patients.

Dans le tiers payant, le contrôle des factures relève des assurances, réduisant la possibilité d'intervenir pour le patient

Le tiers payant tue dans l'œuf l'incitatif souhaité par la Confédération en faveur du contrôle des coûts et de la qualité par le patient. En effet, qui d'autre que le patient est mieux à même de contrôler les factures de médecin? Contre toute attente de la part de santésuisse, une enquête menée par ses soins a indiqué que $70 \%$ des patients contrôlaient les factures de leurs médecins.

Il ne faut pas non plus oublier que tous les médecins qui gèrent leurs factures selon le système du tiers garant font preuve d'une parfaite transparence vis-àvis de leurs patients.

Protection des données et protection contre le bradage du secret médical compromises par le tiers payant

Avec le tiers payant, l'assureur a accès à tous les diagnostics et à toutes les maladies du patient car ils sont inscrits sur toutes les factures qui lui sont adressées.

Dans ce contexte, la facturation mentionnant les positions d'un tarif TARMED extrêmement précis et différencié expose le patient aux limites du secret médical. En effet, les positions détaillées sur la facture permettent d'identifier sans aucun problème le 
diagnostic ainsi que la maladie du patient et d'établir un pronostic sur son état de santé. Le Préposé fédéral à la protection des données a plusieurs fois pointé du doigt ce problème dans ses rapports. Pour le tiers garant, il recommande la gestion des factures de médecins via des centres de confiance.

\section{Tiers garant et échange électronique de données via les centres de confiance}

Chaque année, les centres de confiance gèrent quelque 19 millions de factures, ce qui représente plus d'un milliard de fichiers de données sauvegardées. Par conséquent, cette variante demeure la plus avantageuse pour l'échange électronique des données car celles-ci sont mises quasiment gratuitement à disposition par les centres de confiance et sont conformes à la protection des données. En revanche, les factures traitées et mises à la disposition des assureurs par des intermédiaires comme MediData engendrent des coûts (environ 2 CHF par facture), des dépenses supplémentaires pour les assureurs qui au final se répercutent sur les primes.

Dans l'intérêt de l'ensemble du corps médical et pour les raisons mentionnées ici, il est primordial que la facturation se poursuive principalement selon le tiers garant en favorisant l'échange électronique via les centres de confiance car, financièrement plus avantageux, ce système respecte la protection des données. C'est aussi la raison pour laquelle la FMH continuera d'optimiser les modalités concernant la facturation via les centres de confiance.

\section{Références}

1 Le tiers soldant est une troisième méthode de remboursement prévue par la LAMal (art. 42 al. 1 dernière phrase). On parle de tiers soldant lorsque le patient cède par écrit au fournisseur de prestations le droit d'être remboursé par l'assureur (c.-à-d. ses prétentions nettes, à savoir le montant de la facture après déduction de la franchise et de la quote-part).

2 Art. 42 LAMal:

${ }^{1}$ Sauf convention contraire entre les assureurs et les fournisseurs de prestations, l'assuré est le débiteur de la rémunération envers le fournisseur de prestations. L'assuré a, dans ce cas, le droit d'être remboursé par son assureur (système du tiers garant). En dérogation à l'art. 22, al. 1, LPGA, ce droit peut être cédé au fournisseur de prestations (tiers soldant [note de l'auteur]).

${ }^{2}$ Assureurs et fournisseurs de prestations peuvent convenir que l'assureur est le débiteur de la rémunération (système du tiers payant). En cas de traitement hospitalier, l'assureur, en dérogation à l'al. 1, est le débiteur de sa part de rémunération.

3 Art. 42 al. 1 LAMal (dernière phrase): En dérogation à l'art. 22, al. 1, LPGA, ce droit peut être cédé au fournisseur de prestations.

\title{
Prendre le pouls de l'actualité.
} L'offre groupée de la FMH.

\author{
Bulletin des médecins suisses, Today's Press, \\ FMH-Flash. Gratuit pour nos membres.
}

